\title{
Evidence for Association of the E23K Variant of KCNJ11 Gene with Type 2 Diabetes in Tunisian Population: Population-Based Study and Meta-Analysis
}

\author{
Khaled Lasram, ${ }^{1,2}$ Nizar Ben Halim, ${ }^{1,2}$ Sana Hsouna, ${ }^{1,2}$ Rym Kefi, ${ }^{1,2}$ \\ Imen Arfa, ${ }^{1,2}$ Welid Ghazouani, ${ }^{1,2}$ Henda Jamoussi, ${ }^{1,3}$ Houda Benrahma, ${ }^{4}$ Najla Kharrat, ${ }^{5}$ \\ Ahmed Rebai, ${ }^{5}$ Slim Ben Ammar, ${ }^{2,6}$ Sonia Bahri, ${ }^{2,6}$ Abdelhamid Barakat, ${ }^{4}$ \\ Abdelmajid Abid, ${ }^{1,3}$ and Sonia Abdelhak ${ }^{1,2}$ \\ ${ }^{1}$ Pasteur Institute of Tunis, Laboratory of Biomedical Genomics and Oncogenetics, LR11IPT05, BP 74, 13 Place Pasteur, \\ Le Belvédère, 1002 Tunis, Tunisia \\ ${ }^{2}$ Université Tunis El Manar, 1068 Tunis, Tunisia \\ ${ }^{3}$ National Institute of Nutrition and Food Technology, Department of External Consultation, 11 rue Jebel Lakhdar, \\ Bab Saadoun, 1007 Tunis, Tunisia \\ ${ }^{4}$ Pasteur Institute of Morocco, Department of Scientific Research, Laboratory of Molecular and Human Genetics, \\ 1 Place Louis Pasteur, 20360 Casablanca, Morocco \\ ${ }^{5}$ Centre of Biotechnology of Sfax, Unit of Bioinformatics and Biostatistics, 3038 Sfax, Tunisia \\ ${ }^{6}$ Pasteur Institute of Tunis, Central Laboratory of Medical Biology, 13 Place Pasteur, Le Belvédère, 1002 Tunis, Tunisia \\ Correspondence should be addressed to Khaled Lasram; khaledlas@yahoo.fr
}

Received 26 February 2014; Accepted 30 May 2014; Published 7 July 2014

Academic Editor: Konstantinos Kantartzis

Copyright (C) 2014 Khaled Lasram et al. This is an open access article distributed under the Creative Commons Attribution License, which permits unrestricted use, distribution, and reproduction in any medium, provided the original work is properly cited.

\begin{abstract}
Aims. Genetic association studies have reported the E23K variant of KCNJ11 gene to be associated with Type 2 diabetes. In Arab populations, only four studies have investigated the role of this variant. We aimed to replicate and validate the association between the E23K variant and Type 2 diabetes in Tunisian and Arab populations. Methods. We have performed a case-control association study including 250 Tunisian patients with Type 2 diabetes and 267 controls. Allelic association has also been evaluated by 2 metaanalyses including all population-based studies among Tunisians and Arabs (2 and 5 populations, resp.). Results. A significant association between the E23K variant and Type 2 diabetes was found $(\mathrm{OR}=1.6,95 \% \mathrm{CI}=1.14-2.27$, and $P=0.007)$. Furthermore, our meta-analysis has confirmed the significant role of the E23K variant in susceptibility of Type 2 diabetes in Tunisian and Arab populations $\left(\mathrm{OR}=1.29,95 \% \mathrm{CI}=1.15-1.46\right.$, and $P<10^{-3}$ and $\mathrm{OR}=1.33,95 \% \mathrm{CI}=1.13-1.56$, and $P=0.001$, resp. $)$. Conclusion. Both case-control and meta-analyses results revealed the significant association between the E23K variant of KCNJ11 and Type 2 diabetes among Tunisians and Arabs.
\end{abstract}

\section{Introduction}

Type 2 diabetes is a polygenic disorder characterized by defects in insulin secretion and peripheral insulin resistance [1]. Tunisia, as many countries worldwide, is increasingly affected by diabetes. In the Tunisian population, the prevalence of Type 2 diabetes mellitus (T2DM) reaches 9\% among adults [2] against $2.3 \%$ in 1977 [3]. These values showed an intermediate level compared to several human populations but they are still higher compared to European populations. The increased level of T2DM may be due to the rapid and recent lifestyle changing and/or a specific genetic background.

It is well established that the potassium inwardly rectifying channel, subfamily J, member 11 (KCNJ11) gene located at chromosome 11p15.1 is involved in insulin secretion in humans and that mutations in this KCNJ11 gene can cause 
TABLE 1: Clinical and biochemical characteristics of study subjects.

\begin{tabular}{|c|c|c|c|}
\hline Characteristics & $\mathrm{T} 2 \mathrm{DM}(n=250)$ & Control $(n=267)$ & $P$ value \\
\hline Gender (men/women) & $94 / 156$ & $105 / 162$ & $0.65^{*}$ \\
\hline Age (years) & $60.2 \pm 10.5$ & $53.7 \pm 11.6$ & $<10^{-4^{\dagger}}$ \\
\hline Duration of diabetes (years) & $14.31 \pm 9.26$ & - & - \\
\hline Age at diagnosis (years) & $45.6 \pm 9.5$ & - & - \\
\hline $\mathrm{HbA}_{\mathrm{lc}}(\%(\mathrm{mmol} / \mathrm{mol}))$ & $9.1 \pm 1.9(76 \pm 20)$ & - & - \\
\hline Height $(\mathrm{cm})$ & $161.9 \pm 9.7$ & $162.24 \pm 9.6$ & $0.71^{\dagger}$ \\
\hline Weight (kg) & $75.4 \pm 13.7$ & $74 \pm 13.6$ & $0.3^{\dagger}$ \\
\hline Mean BMI $\left(\mathrm{kg} / \mathrm{m}^{2}\right)$ & $28.9 \pm 5.5$ & $28.2 \pm 5.3$ & $0.2^{\dagger}$ \\
\hline Systolic blood pressure (mmHg) & $14.5 \pm 2$ & $13.4 \pm 2.2$ & $<10^{-4^{\dagger}}$ \\
\hline Diastolic blood pressure (mmHg) & $8.3 \pm 1.1$ & $8.1 \pm 5.2$ & $0.75^{\ddagger}$ \\
\hline Fasting plasma glucose $(\mathrm{mmol} / \mathrm{L})$ & $11.5 \pm 4.1$ & $5.2 \pm 0.5$ & $<10^{-4^{\ddagger}}$ \\
\hline Total cholesterol $(\mathrm{mmol} / \mathrm{L})$ & $5.0 \pm 1.1$ & $5.0 \pm 1$ & $0.95^{\dagger}$ \\
\hline Triglycerides (mmol/L) & $1.7 \pm 0.9$ & $1.3 \pm 0.6$ & $<10^{-4^{\ddagger}}$ \\
\hline HDL-cholesterol (mmol/L) & $1.2 \pm 0.4$ & $1.5 \pm 0.4$ & $<10^{-4^{\dagger}}$ \\
\hline LDL-cholesterol (mmol/L) & $2.8 \pm 0.9$ & $3 \pm 0.9$ & $0.27^{\dagger}$ \\
\hline
\end{tabular}

Data are expressed as means \pm SD (standard deviation). ${ }^{*}$ Pearson's chi-square test. ${ }^{\dagger}$ Student's $t$-test for equal variances. ${ }^{\ddagger}$ Welch's $t$-test for unequal variances.

congenital hyperinsulinism [4] and permanent neonatal diabetes [5]. Consequently, KCNJ11 has been so far investigated as T2DM candidate gene.

In 2003, the E23K variant was shown to be a robustly associating T2DM susceptibility variant [6] and, in 2005, the relationship between this variant and T2DM has been fully elucidated. In fact, KCNJ11 gene encodes a pore-forming subunit of the inwardly rectifying ATP-sensitive $\mathrm{K}+$ channel (Kir6.2), which is one component of the ATP-sensitive potassium $\left(\mathrm{K}_{\mathrm{ATP}}\right)$ channels in pancreatic $\beta$-cells. $\mathrm{K}_{\mathrm{ATP}}$ channels regulate insulin secretion by coupling the metabolic state of the cell to membrane potential. Elevation of blood glucose level leads to an increase in the ATP to ADP ratio and a decrease in $\mathrm{K}_{\mathrm{ATP}}$ channel permeability that in turn leads to membrane depolarization, activation of voltage-dependent calcium channels, $\mathrm{Ca}^{2+}$ influx into the cell, and finally insulin exocytosis [7].

However, the reported functional studies are not yet categorical and the full mutational mechanism(s) is still incomplete [8] due to the difficulty in identifying the causal variant. This is particularly the case when a second nonsynonymous variant in the neighboring ABCC8 gene (rs757110) was found in complete linkage disequilibrium with the E23K variant [9].

The E23K variant has been reported to be associated with T2DM in various ethnic populations, including Europeandescent populations [6, 9, 10] and Asians [11-13]. Among Arab populations, the results were controversial [19-22]. However, these findings cannot be extrapolated to other populations particularly in the Tunisian population which is made of a mosaic of communities and described as genetically heterogeneous.
Thus, we proposed in this study to investigate the role of the E23K variant of KCNJ11 gene in the development of T2DM in the Tunisian population.

\section{Methods}

Details of genotyping data for each subject are given in Table 1. Written informed consent was obtained from all subjects.

2.1. Study Population. The study population consisted of 250 unrelated Tunisian patients with T2DM (62.4\% females; average age at recruitment $60.2 \pm 10.5$ years; average age at T2DM diagnosis $45.6 \pm 9.5$ years) and 267 control subjects (60.7\% females; average age at recruitment $53.7 \pm 11.6$ years) (Table 1).

Subjects with T2DM were diagnosed according to the World Health Organization (WHO) criteria [14] and recruited from National Institute of Nutrition and Food Technology (INNTA), the referral diabetes Medical Center in Tunisia. Subjects with positive glutamic acid decarboxylase antibodies were excluded.

Population control subjects were chosen (1) older than 40 years, age described as significantly related to diabetes in Tunisian population [2], (2) without first degree family history of diabetes given the high familial aggregation within this category compared to the second degree relatives [15], and (3) with normal $(<6.1 \mathrm{mmol} / \mathrm{L})$ fasting plasma glucose.

This study is a part of an international cooperative project, NEPAD/NABNet_T2D_NA (New Partnership for Africa's Development, North Africa Biosciences Network, Type 2 diabetes, North Africa), which aims to assess the involvement of the E23K variant of KCNJ11 with T2DM in four North African 
populations (Tunisia, Algeria, Mauritania, and Morocco). In the present work, we have evaluated the association of the E23K variant in $250 \mathrm{~T} 2 \mathrm{DM}$ patients and 250 controls from Tunisian population.

\subsection{Genetic Analyses}

2.2.1. Blood Sample Collection and DNA Extraction. Peripheral blood was drawn from T2DM patients and controls in ethylenediaminetetraacetic acid (EDTA) anticoagulated tubes. DNA was extracted from the blood using salting-out protocol [16].

2.3. Genotyping. Genotyping of E23K variant was carried out using a TaqMan allelic discrimination assay. The assay was carried out using an ABI Prism 7500 Sequence Detection System (Applied Biosystems, Foster City, CA, USA) in a reaction volume of $20 \mu \mathrm{L}$, according to the manufacturer's instructions and using commercially available primers and probes. A random sample set of $10 \%$ was retested with the same method to confirm genotype accuracy. The genotyping success rate was $97.13 \%$ for cases and controls and the concordance rates were $100 \%$ in duplicate samples.

2.4. Statistical Analysis. Hardy-Weinberg equilibrium (HWE) analyses were performed on $R$ using SNP-HWE program [17]. Comparisons between cases and controls for quantitative traits were performed using Student's $t$-test. For qualitative traits we used chi-square test. We tested E23K variant allelic and genotypic association with T2DM risk using multivariate logistic regression to adjust for age, gender, and body mass index (BMI). Odd ratios (ORs) with 95\% confidence interval ( $95 \%$ CI) were assessed for the risk allele. For the genotyping data we tested various genetic models including additive, dominant, and recessive models. Allelic exact test was computed on $R$ using allelic package. Analysis of variance (ANOVA) or the unpaired two-tailed Student's $t$-test was performed to compare clinical data. Multivariate linear regression was used to test the association of the E23K variant with quantitative traits. A $t$-stat of greater than 1.96 with significance less than 0.05 indicates that the independent variable is a significant predictor of the dependent variable. Statistical analyses were performed using Stata 11 software (StataCorp, College Station, TX, USA). A P value $<0.05$ was considered statistically significant. Power calculations were performed using PS (Power and Sample Size Calculations software (version 3.0)) [18]. Based on probability of exposure in controls obtained in our study (0.19), we had $56.9 \%$ power to detect ORs of 1.68 at $P<0.05$.

2.5. Meta-Analysis. We further collected data from the literature for E23K variant in the KCNJ11 gene in Arab populations by searching PubMed using key words: KCNJ11, Arab, E23K, and Diabetes. Four studies were identified after literature search. Three reports were combined to our study data as the last report by Mtiraoui et al. [20] was a replication of the previous study reported by Ezzidi et al. [19] by increasing the sample size. As the study by Mtiraoui did not report genotyping data, we performed an allelic meta-analysis. Statistical heterogeneity between studies was tested by chisquare-based $Q$ test and quantified using $I$-squared test. The fixed-effects method of Mantel-Haenszel was used when $P_{\text {heterogeneity }}>0.10$ or $I^{2}<50 \%$. Otherwise, a random-effects model of DerSimonian and Laird was used. The conservative Egger's regression analysis was used to evaluate publication bias between the considered 3 studies. Begg's test was used when just two studies are pooled. All statistical analyses were conducted by using STATA software version 11.0.

\section{Results}

3.1. Case-Control Study. Genotypic distribution of E23K did not show any deviation from Hardy-Weinberg equilibrium, $P=0.32$ in T2DM patients and $P=0.69$ in controls (Table 2). The observed clinical and biochemical data are shown in Table 1. The mean age of the diabetic group $(60.2 \pm 10.5)$ was higher $\left(P<10^{-4}\right)$ than that observed among controls $(53.7 \pm 11.76)$. Systolic blood pressure, fasting glucose, and triglycerides means were significantly higher in T2DM patients than in controls $\left(P<10^{-4}\right)$. The high density lipoprotein-cholesterol (HDL-C) distribution was significantly higher in controls than in T2DM patients $(P<$ $\left.10^{-4}\right)$. Sex ratio did not significantly differ between the two groups $(P=0.65)$.

The frequency of the $\mathrm{K}$ allele is significantly higher in cases than in controls (25.4\% versus $19 \% ; P=0.015)$. The $\mathrm{K}$ allele is significantly associated with susceptibility to T2DM (OR $=1.44,95 \% \mathrm{CI}=1.07-1.93$, and $P=0.017$ ) (Table 2$)$.

Logistic regression analysis adjusted for age, gender, and BMI shows a significant association between the E23K variant and T2DM $(\mathrm{OR}=1.6,95 \% \mathrm{CI}=1.14-2.27$, and $P=0.007)$ (Table 2). The E23K variant increased the risk of T2DM in the additive model ( $\mathrm{EE}$ versus $\mathrm{KK}, \mathrm{OR}=3.68,95 \% \mathrm{CI}=1.28-$ 10.58 , and $P=0.016)$, in the dominant model $(\mathrm{OR}=1.61,95 \%$ $\mathrm{CI}=1.07-2.42$, and $P=0.022)$, and in the recessive model $(\mathrm{OR}=3.23,95 \% \mathrm{CI}=1.13-9.21$, and $P=0.028)$ (Table 2$)$.

3.2. Association of E23K Variant with Quantitative Traits. When examining the association between the E23K variant and various T2DM-related quantitative traits (fasting plasma glucose, BMI, total cholesterol, triglycerides, HDL-C, systolic blood pressure, diastolic blood pressure, and low density lipoprotein-cholesterol (LDL-C)) among T2DM group, control group, and the overall sample, fasting plasma glucose values were found to differ significantly across the E23K genotypes in T2DM and in the pooled sample, $P=0.04$ and $P=0.03$, respectively (Table 3 ). Fasting plasma glucose levels were higher in subjects with the EK genotype $(12.49 \pm 4.13)$ than in those with the KK $(10.81 \pm 4.48)$ and EE genotypes $(11.03 \pm 3.89)$ in T2DM group. When considering the overall sample, a different tendency was observed and fasting plasma glucose levels were higher in subjects with the KK genotype $(9.54 \pm 4.53)$ than in those with the EK $(9.32 \pm 4.81)$ and EE genotypes $(7.93 \pm 3.93)$. However, no significant differences were observed between genotypes and groups for BMI, total 


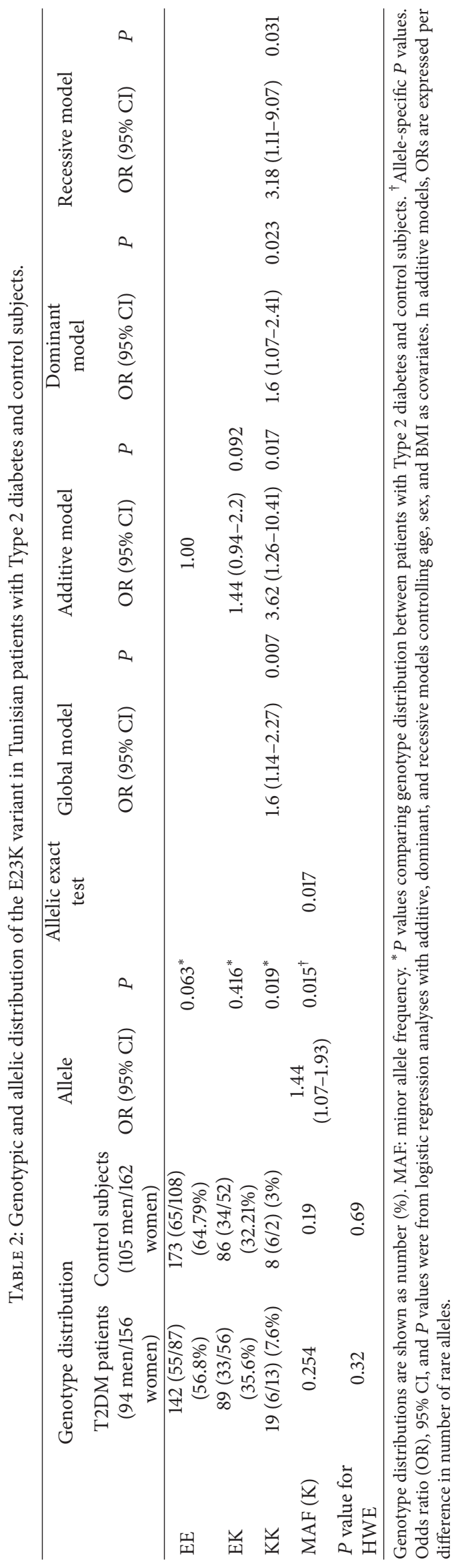




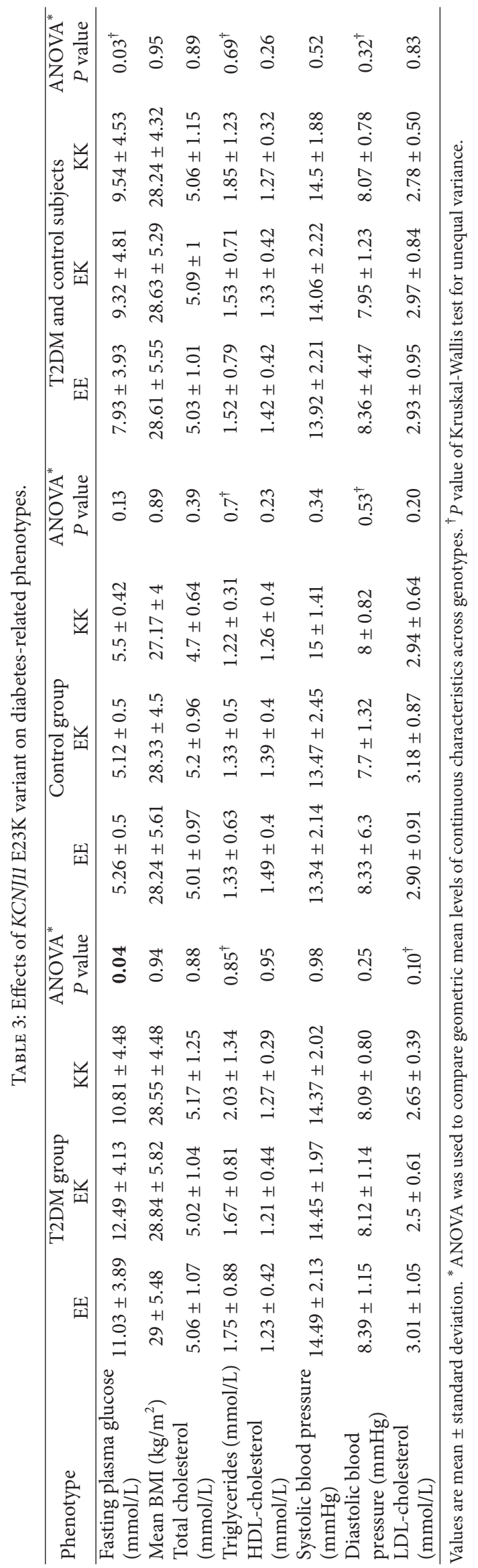


TABLE 4: Results of multiple regression analysis under a dominant model.

\begin{tabular}{lcccc}
\hline Dependent variable & Independent variables & Stand beta & $t^{*}$ & $P$ value $^{*}$ \\
\hline & KCNJ11 E23K genotype & 0.65 & 2.17 & 0.031 \\
Fasting plasma glucose $(\mathrm{mmol} / \mathrm{L})$ & Gender & 0.03 & 0.09 & 0.926 \\
& Age & -0.007 & -0.55 & 0.585 \\
& BMI & 0.003 & 0.12 & 0.907 \\
& T2DM (presence or absence) & 6.43 & 21.65 & $P<10^{-3}$ \\
\hline
\end{tabular}

${ }^{*}$ Stand beta standardized beta-coefficient; ${ }^{\dagger} t$-statistic; ${ }^{\ddagger} P$ value for the $t$-statistic.

cholesterol, triglycerides, HDL-C, systolic blood pressure, and diastolic blood pressure.

Multiple regression analyses were performed to assess the independent contribution of the statute (presence or absence of T2DM), the age, the gender, and the BMI to fasting plasma glucose. E23K genotype of the KCNJ11 gene was found to be independently related to fasting plasma glucose levels under a dominant model: fasting plasma glucose explained 65\% $(P=$ 0.031 ) of the E23K variability of E23K (Table 4).

3.3. Tunisian Meta-Analysis. We first pooled overall effects of the E23K variant and T2DM risk of 1720 Tunisian patients and 1105 control subjects from the current study with the one from Central East Tunisia of Mtiraoui et al. [20]. The pooled OR for allelic frequency comparison suggested that the E23K variant is significantly associated with an increased T2DM risk: $\mathrm{OR}=1.29,95 \% \mathrm{CI}=1.15-1.46, P<10^{-3}, P_{\text {heterogeneity }}=$ 0.427 , and $I^{2}=0.0 \%$ (fixed effects) (Figure 1). Begg's test showed that there is no publication bias for E23K variant $(P=0.317)$.

3.4. Arab Meta-Analysis. We extended the analysis to Arab populations and performed a meta-analysis of the E23K variant on 3156 T2DM patients and 2493 controls. For this purpose, we have added results from a Saudi study realized in 2007 [21], the Lebanese participants of the Mtiraoui et al. study [20], and a recent Mauritanian study [22] to the two previous Tunisian samples.

The pooled OR for allelic frequency comparison suggested that the $\mathrm{E} 23 \mathrm{~K}$ variant is significantly associated with an increased T2DM risk: $\mathrm{OR}=1.33,95 \% \mathrm{CI}=1.13-1.56, P=$ $0.001, P_{\text {heterogeneity }}=0.03$, and $I^{2}=62.7 \%$ (random effects) (Figure 2). Egger's test showed that there is no publication bias (Egger's test) for E23K variant $(P=0.228)$.

\section{Discussion}

In the present study, we sought to replicate the association of the E23K variant in KCNJ11 gene identified in the aforementioned studies $[6,9,10,20-22]$ with T2DM in an independent Tunisian case-control sample. We validated the positive association between E23K and T2DM in the Tunisian population with an increased risk of 1.6. Interestingly, T2DM subjects with the EK genotype had more severe hyperglycemia than subjects with EE or KK. Likewise, controls carrying the KK genotype had the highest fasting plasma glucose level, suggesting genotype-phenotype correlation as reported by Schwanstecher et al. [23]. In the former study, the heterozygous (EK) and homozygous (KK) state of E23K variant promote the development of T2DM by increasing the ATP concentration threshold that induces both pancreatic $\beta$-cells $\mathrm{K}_{\mathrm{ATP}}$ channels overactivity and insulin secretion inhibition [23]. Nielsen et al. [10] have also reported the association of E23K variant with decreased levels of insulin secretion in nondiabetic individuals.

We also found that the KK genotype confers a 3.62 times higher risk for T2DM compared to the EE genotype. Our findings were in contrast with several studies on Caucasians $[6,9,10]$ and Chinese Han [13] showing a significant association between the K23 allele and T2DM under a recessive model. Our results were also not in accordance with some Asian studies on Japanese [11] and Korean populations [12], where a strong association has been reported between the K23 allele and T2DM under a dominant model. Few studies were performed on the involvement of the E23K variant on T2DM pathogenesis in Arab populations. Alsmadi et al. [21] have shown a significant association of the K23 allele under an additive model (EE versus EK) while it has been reported under a dominant model in the Mauritanian population [22]. These controversial results show specific ethnic differentiation of the E23K variant effect under different genetic models. In contrast to results found in Mtiraoui et al. [20], study on population from Central East Tunisia revealed no significant association [19]. This discrepancy might be explained by the population stratification in the Central East of Tunisia. Indeed, a replication study with a larger sample size showed a positive association [20]. The former study reported no significant association in the Lebanese population [20]. In our studied sample, the $\mathrm{K}$ minor allele frequency (MAF) of the E23K variant was 0.25 in cases and 0.19 in controls (Table 2), while in Mtiraoui et al., it was found at 0.33 in cases and 0.28 in controls [20]. Similarly, in Alsmadi et al., the MAF of E23K variant was 0.21 in cases and 0.14 in controls [21]; in Abdelhamid et al., it was of 0.22 in cases and 0.16 in controls [22]. In Caucasian populations, such as the Danish and British, the MAF of the $\mathrm{E} 23 \mathrm{~K}$ variant seems to be more homogenous among cases and controls with a frequency of 0.4 and 0.38 in the Danish [10] and 0.4 and 0.36 in the British, respectively [6].

Interestingly, all these data showed that allelic distribution in our sample is closer to Saudi and Mauritanian populations than to the East-Central Tunisians. These findings reveal the genetic heterogeneity of the Tunisian population which is made of a mosaic of communities from different ethnic background (Arab, Berber, Roman, etc.). This 


\begin{tabular}{|c|c|c|c|}
\hline \multicolumn{2}{|l|}{$\begin{array}{l}\text { Study } \\
\text { ID }\end{array}$} & \multicolumn{2}{|r|}{$(\%)$} \\
\hline & & OR (95\% CI) & Weight \\
\hline Mtiraoui, et al. (2012) Tunisians & $\begin{aligned} 1 \\
-1\end{aligned}$ & $1.27(1.11,1.44)$ & 84.46 \\
\hline Present study (2014) & 1 & $1.44(1.07,1.94)$ & 15.54 \\
\hline Overall $\left(I^{2}=0.0 \%, P=0.427\right)$ & & $1.29(1.15,1.46)$ & 100.00 \\
\hline 0.5 & & 2 & \\
\hline
\end{tabular}

Figure 1: Meta-analysis of the association between KCNJ11 E23K and T2DM in Tunisian population. Forest plot of allelic odds ratio (OR). The area of the squares reflects the study specific weight. The diamond shows the summary fixed-effects odds ratio estimate from 2 studies.

\begin{tabular}{|c|c|c|c|}
\hline Study & & & $(\%)$ \\
\hline ID & & OR $(95 \% \mathrm{CI})$ & Weight \\
\hline Alsmadi, et al. (2008) Saudi & & $1.69(1.30,2.20)$ & 18.25 \\
\hline Mtiraoui, et al. (2012) Tunisians & $\rightarrow$ & $1.27(1.11,1.44)$ & 28.65 \\
\hline Mtiraoui, et al. (2012) Lebanese & $\rightarrow$ & $1.07(0.92,1.25)$ & 26.86 \\
\hline Abdelhamid, et al. (2014) Mauritanian & & $1.52(0.98,2.35)$ & 9.92 \\
\hline Present study (2014) & & $1.44(1.07,1.94)$ & 16.32 \\
\hline Overall $\left(I^{2}=62.7 \%, P=0.030\right)$ & & $1.33(1.13,1.56)$ & 100.00 \\
\hline Note: weights are from random-effects analysis & & & \\
\hline
\end{tabular}

FIGURE 2: Meta-analysis of the association between KCNJ11 E23K and T2DM in Arab population. Forest plot of allelic odds ratio (OR) under the random-effects model. The area of the squares reflects the study specific weight. The diamond shows the summary odds ratio estimate from 5 studies.

population stratification was additionally maintained by a high prevalence of endogamous unions [24]. The observed MAF similarities between the Tunisian and Saudi might be explained by the Arab conquest in Tunisia that started in the 7 th century, followed by a massive Bedouin invasion during the 11th century [25] that has largely contributed to the Tunisian gene pool. In the same context, the Mauritanian study performed by Abdelhamid et al. [22] explained the similarity of their K minor allele frequencies with the Saudi by the genetic affinities between the Moor group (80\% of Mauritanians) and Arabs [22, 26].

On the other hand, findings from Mtiraoui et al. [20] showed that East-Central Tunisians are most related to Caucasians. These genetic similarities probably correspond to the well-known historical European influence in this region $[27,28]$, thus explaining the variability of the genetic background between the coastal regions of central Tunisia and the rest of the country. In this context, one study on the mitochondrial DNA diversity of the population of
Monastir showed the relative predominance of Eurasian haplogroups (63\%) in the East-Central areas of Tunisia (unpublished data).

When performing a "Tunisian" meta-analysis (Figure 1), the E23K variant was still associated with a 1.29-fold increased risk of T2DM $\left(P<10^{-3}\right)$. We have also performed allelic meta-analysis in Arab population given the fact that several meta-analyses have reported the association of E23K with an increased T2DM risk in Caucasians and Asians [6, 9-11, 13, 29]. We found that the E $23 \mathrm{~K}$ variant is strongly associated with T2DM. Our results are consistent with previous metaanalyses among different ethnic groups where the E23K variant is considered to be a strong candidate variant for T2DM worldwide. In this context, two Caucasian metaanalyses $[10,29]$, grouping 4 studies (2824 subjects) and 18 studies (7768 subjects), respectively, reported similar results, with a significant association with T2DM, under a recessive model $(\mathrm{OR}=1.49, P=0.00022)$ and additive model $(\mathrm{OR}=$ 1.44, $P=0.0007)$, respectively. Likewise, two East Asian 
meta-analyses conducted by Zhou et al. [13] and by Takeuchi et al. [30] grouping, respectively, 8 studies (15503 subjects) and 7 studies (8217 subjects) showed association with an OR of $1.15\left(P=3 \times 10^{-9}\right)$ and an OR of $1.168\left(P<10^{-3}\right)$, respectively. More recently, a global meta-analysis based on 49 case-control studies [31] reported a significant association with T2DM under a random-effects model, with per-allele odds ratio of $1.13\left(P<10^{-5}\right)$. Significant results were found in only East Asians and Caucasians after ethnic stratification. Unfortunately, in the former study, the association in Arab populations has not been tested according to their ethnicity.

In conclusion, our case-control study confirmed that the KCNJ11 E23K variant is significantly associated with the susceptibility to T2DM in the Tunisian population. Further genetic and functional studies are required to identify the relevant variant(s) at this locus and to investigate their effect(s) on T2DM susceptibility. To our knowledge, this study represents the first meta-analysis of E23K variant in KCNJ11 gene in association with T2DM in Arab populations and complement previous meta-analyses in Asians and Caucasians. Nevertheless, due to our small sample size, further larger investigations and meta-analysis are needed to confirm the role of the $\mathrm{E} 23 \mathrm{~K}$ variant in genetic predisposition to T2DM in Arab populations.

\section{Conflict of Interests}

The authors declare no conflict of interests.

\section{Authors' Contribution}

Khaled Lasram and Nizar Ben Halim have equally contributed to this work.

\section{Acknowledgments}

This work was supported by NEPAD/NABNet_T2D_NA (New Partnership for Africa's Development, North Africa Biosciences Network, Type 2 diabetes, North Africa), joint WHO/EMRO-COMSTECH RAB\&GH Grants, Tunisian Ministry of Higher Education and Scientific Research, and Tunisian Ministry of Health.

\section{References}

[1] J. E. Gerich, "The genetic basis of type 2 diabetes mellitus: impaired insulin secretion versus impaired insulin sensitivity," Endocrine Reviews, vol. 19, no. 4, pp. 491-503, 1998.

[2] R. Bouguerra, H. Alberti, L. B. Salem et al., "The global diabetes pandemic: the Tunisian experience," European Journal of Clinical Nutrition, vol. 61, no. 2, pp. 160-165, 2007.

[3] L. Papoz, N. Achour, M. Henry-Amar, F. Ben Khalifa, E. Eschwege, and H. Ben Ayed, "Cross-sectional study of growth between 3 and 20 years in the population of Tunis," Revue d'Epidemiologie et de Sante Publique, vol. 31, no. 1, pp. 1-10, 1983.

[4] C. James, R. R. Kapoor, D. Ismail, and K. Hussain, “The genetic basis of congenital hyperinsulinism," Journal of Medical Genetics, vol. 46, no. 5, pp. 289-299, 2009.
[5] J. V. Sagen, H. Ræder, E. Hathout et al., "Permanent neonatal diabetes due to mutations in KCNJ11 encoding Kir6.2: patient characteristics and initial response to sulfonylurea therapy," Diabetes, vol. 53, no. 10, pp. 2713-2718, 2004.

[6] A. L. Gloyn, M. N. Weedon, K. R. Owen et al., "Large-scale association studies of variants in genes encoding the pancreatic $\beta$ cell $\mathrm{K}_{A T P}$ channel subunits Kir6.2 (KCNJ11) and SUR1 (ABCC8) confirm that the KCNJ11 E23K variant is associated with type 2 diabetes," Diabetes, vol. 52, no. 2, pp. 568-572, 2003.

[7] M. J. Riedel, D. C. Steckley, and P. E. Light, "Current status of the E23K Kir6.2 polymorphism: implications for type-2 diabetes," Human Genetics, vol. 116, no. 3, pp. 133-145, 2005.

[8] K. S. C. Hamming, D. Soliman, L. C. Matemisz et al., "Coexpression of the type 2 diabetes susceptibility gene variants KCNJ11 E23K and ABCC8 S1369A alter the ATP and sulfonylurea sensitivities of the ATP-sensitive $\mathrm{K}^{+}$channel," Diabetes, vol. 58, no. 10, pp. 2419-2424, 2009.

[9] J. C. Florez, N. Burtt, P. I. W. de Bakker et al., "Haplotype structure and genotype-phenotype correlations of the sulfonylurea receptor and the islet ATP-sensitive potassium channel gene region," Diabetes, vol. 53, no. 5, pp. 1360-1368, 2004.

[10] E. M. Nielsen, L. Hansen, B. Carstensen et al., “The E23K variant of Kir6.2 associates with impaired post-OGTT serum insulin response and increased risk of type 2 diabetes," Diabetes, vol. 52, no. 2, pp. 573-577, 2003.

[11] Y. Sakamoto, H. Inoue, P. Keshavarz et al., "SNPs in the KCNJ11$A B C C 8$ gene locus are associated with type 2 diabetes and blood pressure levels in the Japanese population," Journal of Human Genetics, vol. 52, no. 10, pp. 781-793, 2007.

[12] B. K. Koo, Y. M. Cho, B. L. Park et al., "Polymorphisms of KCNJ11 (Kir6.2 gene) are associated with type 2 diabetes and hypertension in the Korean population," Diabetic Medicine, vol. 24, no. 2, pp. 178-186, 2007.

[13] D. Zhou, D. Zhang, Y. Liu et al., "The E23K variation in the KCNJ11 gene is associated with type 2 diabetes in Chinese and East Asian population," Journal of Human Genetics, vol. 54, no. 7, pp. 433-435, 2009.

[14] K. G. Alberti and P. Z. Zimmet, "Definition, diagnosis and classification of diabetes mellitus and its complications. Part 1: diagnosis and classification of diabetes mellitus provisional report of a WHO consultation," Diabetic Medicine, vol. 15, no. 7, pp. 539-553, 1998.

[15] I. Arfa, A. Abid, D. Malouche et al., "Familial aggregation and excess maternal transmission of type 2 diabetes in Tunisia," Postgraduate Medical Journal, vol. 83, no. 979, pp. 348-351, 2007.

[16] S. A. Miller, D. D. Dykes, and H. F. Polesky, "A simple salting out procedure for extracting DNA from human nucleated cells," Nucleic Acids Research, vol. 16, no. 3, article 1215, 1988.

[17] J. E. Wigginton, D. J. Cutler, and G. R. Abecasis, "A note on exact tests of Hardy-Weinberg equilibrium," The American Journal of Human Genetics, vol. 76, no. 5, pp. 887-893, 2005.

[18] W. D. Dupont and W. D. Plummer Jr., "Power and sample size calculations. A review and computer program," Controlled Clinical Trials, vol. 11, no. 2, pp. 116-128, 1990.

[19] I. Ezzidi, N. Mtiraoui, S. Cauchi et al., "Contribution of type 2 diabetes associated loci in the Arabic population from Tunisia: a case-control study," BMC Medical Genetics, vol. 10, article 33, 2009.

[20] N. Mtiraoui, A. Turki, R. Nemr et al., "Contribution of common variants of ENPP1, IGF2BP2, KCNJ11, MLXIPL, PPAR , $S L C 30 A 8$ and TCF7L2 to the risk of type 2 diabetes in Lebanese 
and Tunisian Arabs," Diabetes and Metabolism, vol. 38, no. 5, pp. 444-449, 2012.

[21] O. Alsmadi, K. Al-Rubeaan, S. M. Wakil et al., "Genetic study of Saudi diabetes (GSSD): significant association of the KCNJ11 E23K polymorphism with type 2 diabetes," Diabetes/Metabolism Research and Reviews, vol. 24, no. 2, pp. 137-140, 2008.

[22] I. Abdelhamid, K. Lasram, G. Meiloud et al., "E23K variant in KCNJ11 gene is associated with susceptibility to type 2 diabetes in the Mauritanian population," Primary Care Diabetes, vol. 8, no. 2, pp. 171-175, 2014.

[23] C. Schwanstecher, U. Meyer, and M. Schwanstecher, " $\mathrm{K}_{\mathrm{IR}} 6.2$ polymorphism predisposes to type 2 diabetes by inducing overactivity of pancreatic $\beta$-cell ATP-sensitive $\mathrm{K}^{+}$channels," Diabetes, vol. 51, no. 3, pp. 875-879, 2002.

[24] S. Riou, C. El Younsi, and H. Chaabouni, "Consanguinity in the population of Northern Tunisia," Tunisie Medicale, vol. 67, no. 3, pp. 167-172, 1989.

[25] A. Hajjej, S. Hmida, H. Kaabi et al., "HLA genes in Southern Tunisians (Ghannouch area) and their relationship with other Mediterraneans," European Journal of Medical Genetics, vol. 49, no. 1, pp. 43-56, 2006.

[26] F. M. Veten, I. O. Abdelhamid, G. M. Meiloud et al., "Hb $\mathrm{S}[\beta 6(\mathrm{~A} 3) \mathrm{Glu} \rightarrow \mathrm{Val}, \mathrm{GAG}>\mathrm{GTG}]$ and $\beta$-globin gene cluster haplotype distribution in Mauritania," Hemoglobin, vol. 36, no. 4, pp. 311-315, 2012.

[27] C. A. Julien, Histoire de l'Afrique du Nord: Des Origines à 1830, Centre d'Etudes et de Recherches Economiques et Sociales (Ceres), Tunisie, Tunisia, 2003.

[28] L'Association Francaise Pour L'Avancement des Sciences, La Tunisie Histoire et Description, Berger-Levrault, Paris, France, 1896.

[29] R. M. van Dam, B. Hoebee, J. C. Seidell, M. M. Schaap, T. W. A. de Bruin, and E. J. M. Feskens, "Common variants in the ATPsensitive $\mathrm{K}^{+}$channel genes KCNJ11 (Kir6.2) and ABCC8 (SUR1) in relation to glucose intolerance: population-based studies and meta-analyses," Diabetic Medicine, vol. 22, no. 5, pp. 590-598, 2005.

[30] M. Takeuchi, K. Okamoto, T. Takagi, and H. Ishii, "Ethnic difference in patients with type 2 diabetes mellitus in interEast Asian populations: a systematic review and meta-analysis focusing on gene polymorphism," Journal of Diabetes, vol. 1, no. 4, pp. 255-262, 2009.

[31] B. Gong, J. Yu, H. Li, W. Li, and X. Tong, "The effect of KCNJ11 polymorphism on the risk of type 2 diabetes: a global meta-analysis based on 49 case-control studies," DNA and Cell Biology, vol. 31, no. 5, pp. 801-810, 2012. 


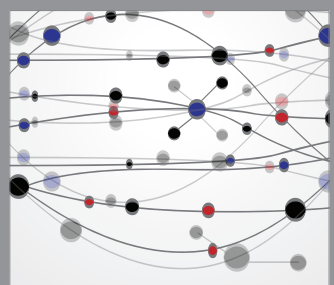

The Scientific World Journal
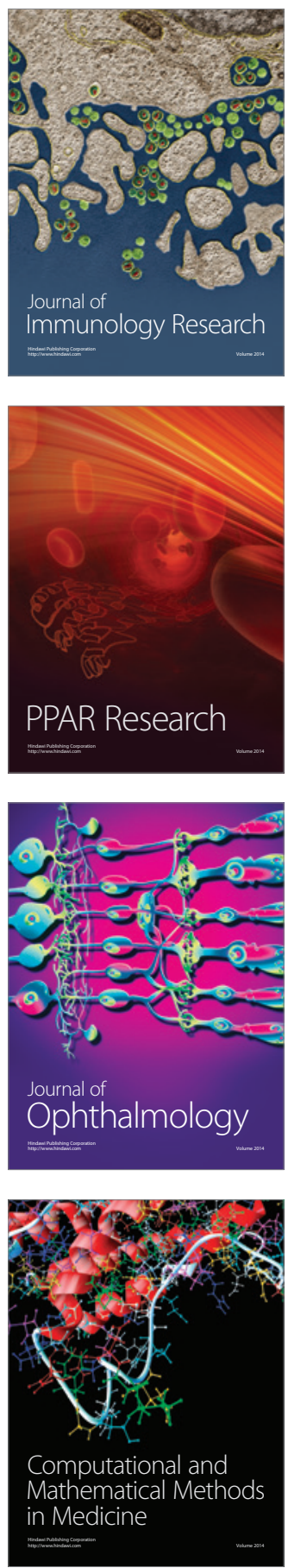

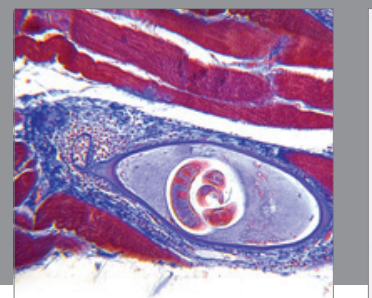

Gastroenterology

Research and Practice
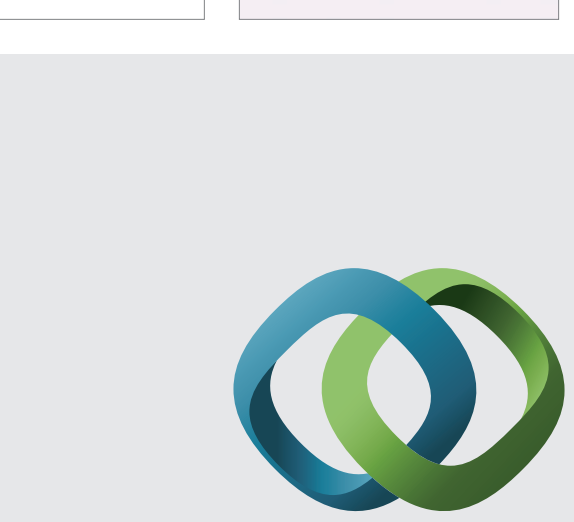

\section{Hindawi}

Submit your manuscripts at

http://www.hindawi.com
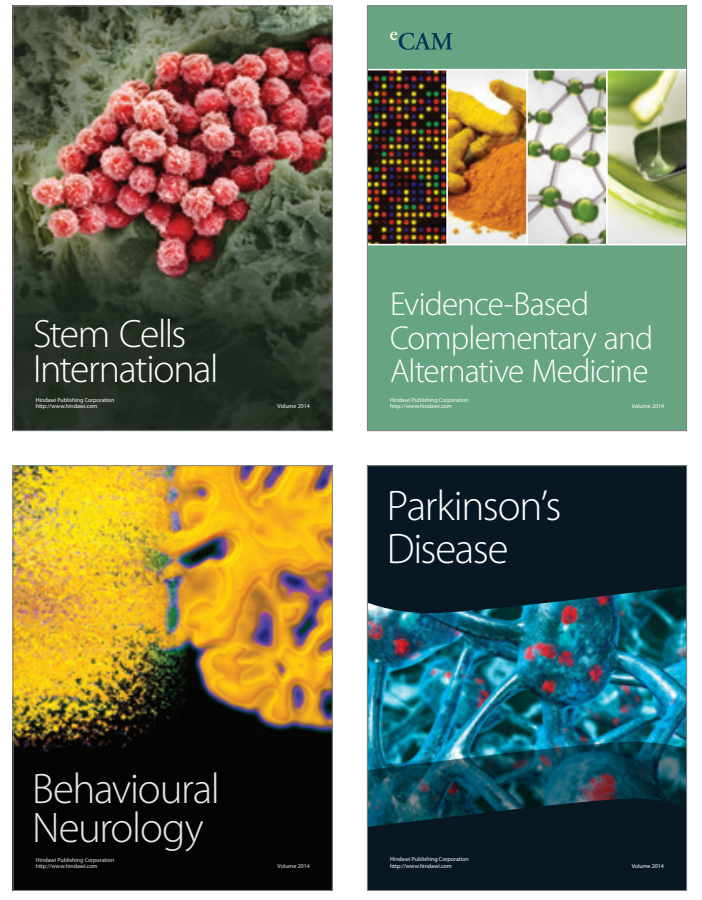
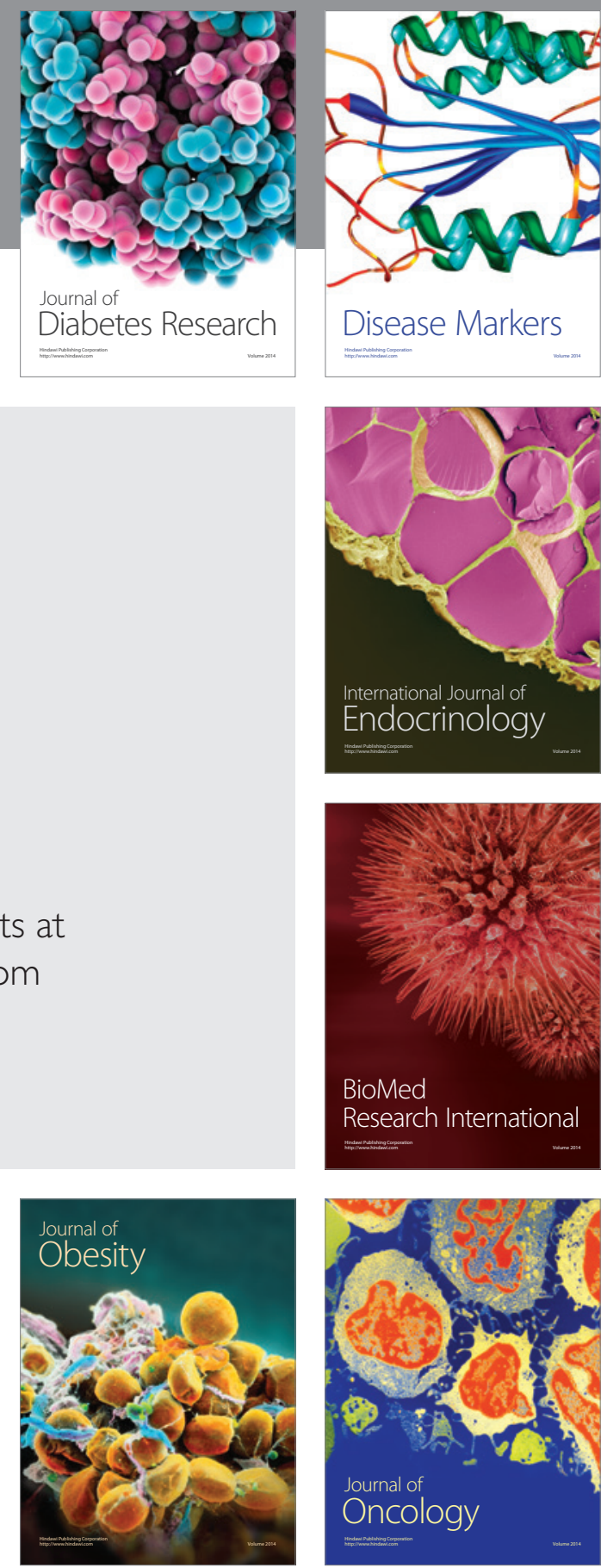

Disease Markers
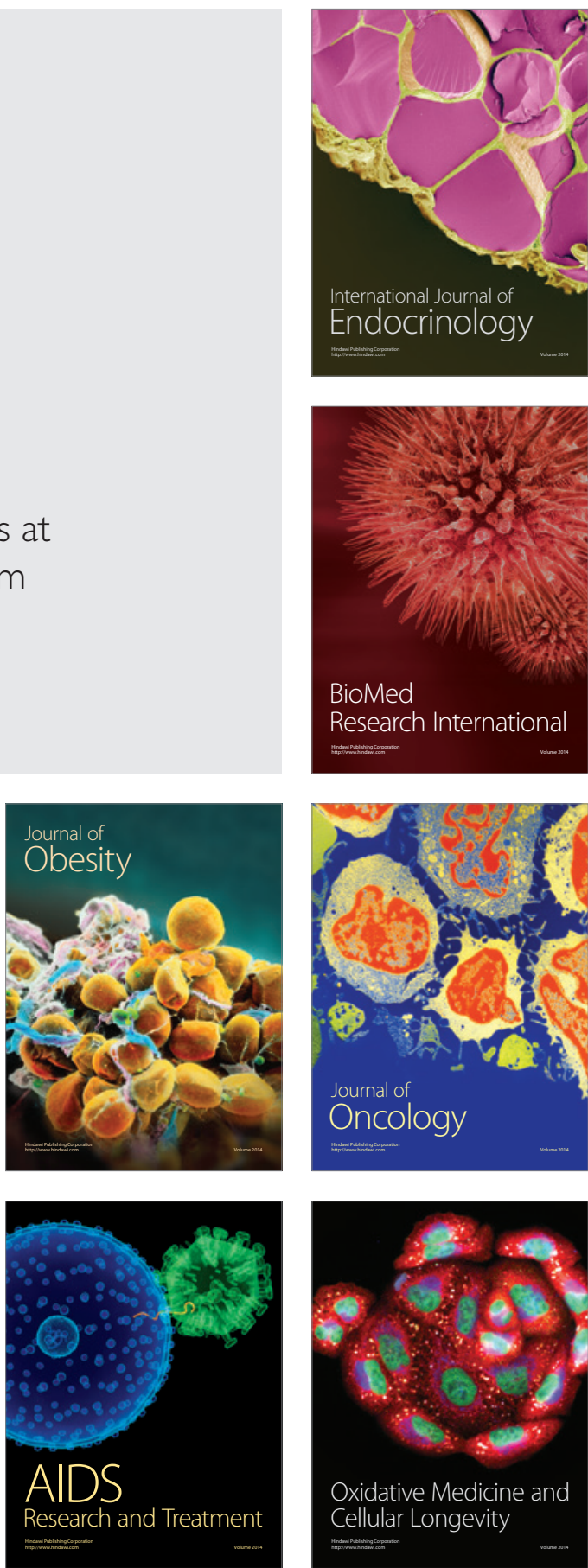